\title{
Nonsteroidal anti-inflammatory drugs in community-acquired pneumonia
}

To the Editor:

We read with interest the editorial by RicCARD and MEDISSA [1], which accompanied the paper by TAGAMI et al. [2] that reviewed the effects of corticosteroids on mortality in community-acquired pneumonia (CAP).

However, we would like to urge caution regarding one of the conclusions drawn by the authors of the editorial, namely that clinicians should refrain from giving nonsteroidal anti-inflammatory drugs (NSAIDs) to patients in the early stages of CAP [1].

The editorial by RicCARD and MEDissa [1] references two studies that have shown "patients exposed to NSAIDs during the early stage of CAP had a worse presentation of CAP, more pleuropulmonary complications and required noninvasive ventilatory support more often, such as high-flow oxygen therapy".

One of the referenced articles, a study conducted by the editorial authors, was a retrospective case note review of 106 patients over a 12-year period, 20 of whom had been prescribed NSAIDs [3]. The explanations offered for the findings were two-fold: 1) that the administration of NSAIDs delayed antibiotic prescription, although this observed trend did not reach statistical significance in their study; and 2) that the NSAIDs might have a direct, dampening effect on patients' immune response, something for which there is little supporting evidence in humans.

The second referenced article was a prospective, but uncontrolled, observational study looking to document the presentation and course of CAP with a particular focus on NSAID use [4]. They found that 32 patients using NSAIDs in the early stages of CAP had a more subacute presentation, evidenced by a delayed referral to hospital and more pleuropulmonary complications, but they had no more severe systemic inflammations than the 58 patients who had not received NSAIDs during their pneumonic episodes.

NSAIDs have not been shown in either of these studies to be the cause of the observed differences in presentation and the disease course of patient episodes of CAP. A more intuitive explanation, not explored by the authors of either paper, is that those patients taking NSAIDs in the context of a pneumonic illness are doing so in order to gain symptomatic relief of pleuritic pain. These patients are likely to be a self-selecting group and at risk of pleural complications as well as suffering from a more severe pneumonia, not because they are taking NSAIDs but because of their requirement for them.

Issuing guidance to general practitioners advising them to avoid NSAIDs in patients with clinical signs suggestive of pneumonia would be an inappropriate interpretation of the available evidence and risks harm by potentially denying patients a powerful analgesic that may be indicated. One should also be wary of extrapolating data from intensive care populations to guide treatment of all patients in the wider community where presentation and the course of the disease are much more variable.

@ERSpublications

Advising against NSAIDs is an inappropriate interpretation of current evidence and may increase patient morbidity http://ow.ly/LAxiu

Emma Crawford, Martin Allen, Naveed Mustfa and Helen Stone

Dept of Respiratory Medicine, Royal Stoke University Hospital, University Hospitals of North Midlands, Staffordshire, UK.

Correspondence: Emma Crawford, Dept of Respiratory Medicine, Royal Stoke University Hospital, University Hospitals of North Midlands, Newcastle Road, Stoke on Trent, ST4 6QG, Staffordshire, UK. E-mail: emma-jane.crawford@uhns.nhs.uk

Received: Feb 252015 | Accepted: March 042015

Conflict of interest: None declared.

\section{References}

1 Ricard J-D, Messika J. Low-dose corticosteroids during severe community-acquired pneumonia: end of the story. Eur Respir J 2015; 45: 305-307.

2 Tagami T, Matsui $\mathrm{H}$, Horiguchi $\mathrm{H}$, et al. Low-dose corticosteroid use and mortality in severe community-acquired pneumonia patients. Eur Respir J 2015; 45: 463-472. 
3 Messika J, Sztrymf B, Bertrand F, et al. Risks of nonsteroidal antiinflammatory drugs in undiagnosed intensive care unit pneumococcal pneumonia: younger and more severely affected patients. J Crit Care 2014; 29: 733-738.

4 Voiriot G, Dury S, Parrot A, et al. Nonsteroidal antiinflammatory drugs may affect the presentation and course of community-acquired pneumonia. Chest 2011; 139: 387-394.

From the authors:

We read with attention the letter by E. Crawford and co-workers and thank them for their interests in our work.

Our retrospective study [1] found that among patients admitted to our intensive care unit (ICU) with pneumococcal community-acquired pneumonia (CAP), we were able to distinguish two distinct populations with respect to exposure to Nonsteroidal anti-inflammatory drugs (NSAIDs). The group of patients who had taken NSAIDs was, surprisingly, much younger (on average 20 years younger) and in a healthier condition than those who had not. Counterintuitively, the much younger and healthier patient group required ICU and organ failure support in a similar manner to those who were sicker and older. We raised the hypothesis that exposure to NSAIDs may have contributed to this situation, possibly by blunting signs and delaying antibiotic prescription. Because our results are in line with other data on the danger of NSAIDs in other infectious settings [2], such as soft tissue infections [3], our conclusion was one of caution regarding the use of NSAID in the context of an infection in progress.

E. Crawford and co-workers argue that our data do not support our conclusion, and that NSAIDs might well have been given because pleural effusion was already present.

They erroneously state that there was no delay in antibiotic prescription. On the contrary, we found that patients exposed to NSAIDs received antibiotics 4.5 days after initial medical consultation, whereas those who did not receive NSAIDs received antibiotics sooner, 2.5 days after initial consultation. Interestingly, time from onset of symptoms to initial consultation was identical in the two groups, suggesting that the degree of severity of symptoms and overall presentation were similar at the start. In an ongoing infectious process, this delay can have serious consequences and may well explain, contrary to E. Crawford and co-workers' hypothesis, the greater pleural involvement in the exposed patients, although we agree our results are not definite proof of NSAIDs' responsibility. We also acknowledge the retrospective nature of our study and its limits.

Nonetheless, NSAIDs are not the innocuous medication E. Crawford and co-workers suggest they are i.e. that they can be widely prescribed in the community. Excess deaths associated with NSAIDs exceed sixteen thousand patients a year in the USA [4] and costs associated with side effects from NSAIDs reaches $€ 59$ million in the Netherlands annually [5].

We are not "extrapolating data from intensive care populations to guide treatment of all patients in the wider community", since the data we gathered came from the community!

And guiding treatment is precisely a key issue! Surprisingly (or not...), NSAIDs do not appear in the USA [6], European [7] or French [8] guidelines on management of CAP and lower respiratory tract infections. They solely appear in the British Thoracic Society guidelines, and only after paracetamol "for symptomatic treatment of pleuritic chest pain of pneumonia treated in the community" [9]. Importantly, paracetamol is recommended with a weak level of evidence (D) but NSAIDs appear without any evidence whatsoever. In addition, use of NSAIDs do not appear in the BTS guidelines for the management of pleural infection [10]. Hence, there is no data to support the use of NSAIDs as an adjunct therapy for CAP.

Diagnosing CAP in the community is not an easy task, especially in young adults. Our concern is that precisely because they are younger and healthier with fewer comorbidities, they receive fewer (or less promptly) antibiotics. Alleviating pain is a necessary and laudable goal but, to date, no study has demonstrated the superiority of NSAIDs over paracetamol in pneumonia-associated pleuritic chest pain, whereas there is ample evidence demonstrating the detrimental consequence of delaying antibiotherapy.

Caution warrants that general practitioners should avoid NSAIDs in patients with clinical signs suggestive of pneumonia. One should be wary of over interpreting inexistent data. 